\title{
A modification of Modified Hausdorff Distance method applying for face recognition
}

\author{
Dang Nguyen Chau and Do Hong Tuan
}

\begin{abstract}
Face recognition, that has a lot of applications in modern life, is still an attractive research for pattern recognition community. Due to the similarity of human face, face recognition presents a significant chalenge for pattern recognition researchers. Modified Hausdorff distance (MHD) is a low computational cost while giving high accuracy for face recognition. In this paper, a modification of MHD (MMHD) is proposed. By applying the ratio of high confident into the calculation of the distance between images, the MMHD gives higher accuracy in face recognition in comparing with MHD method. The MMHD method also gives higher performance than MHD method in face recognition in various nonideal conditions of image: 1) varying lighting conditions, 2) varying face expressions and 3) varying of poses.
\end{abstract}

Index Terms - Edge map, Modified MHD, MMHD, Hausdorff Distance, MHD.

\section{INTRODUCTION}

$\mathrm{D}$ ue to the increasing of the identification applications, the demand for face recognition techniques is increasing day by day. In comparing with other identification techniques, face recognition for identification is the most friendly technique with users. Face recognition is used for identifying one or more persons from a still image or a video by comparing the input image with face images stored in database. Face features of images in database are extracted and stored offline. The same features of the input image are extracted too and compared with the features of each model

Manuscript Received on March 15 ${ }^{\text {th }}$, 2017, Manuscript Revised on November 01 ${ }^{\text {st }}, 2017$.

Dang Nguyen Chau, Ho Chi Minh City University of Technology - VNU-HCM, Ho Chi Minh City, Vietnam (email: chaudn@hcmut.edu.vn).

Do Hong Tuan, Ho Chi Minh City University of Technology - VNU-HCM, Ho Chi Minh City, Vietnam (email: do-hong@hcmut.edu.vn). image in the database. Due to the similarity of human face, comparing techniques of face features still present significant challenge for pattern recognition researchers. Furthermore, light conditions, face expressions and pose variations also make the face recognition more complicate.

For last 30 years, face recognition has been an attractive problem for pattern recognition researchers. Many face recognition methods have been introduced by researcher, which could be categorized in five groups: Eigenface [1], Neural network [2], Graph matching [4], Hidden Markov Model [5], Geometrical feature matching [6].

Edge curves face is one of important features of human face. However, it had been not widely used in face recognition techniques. MHD, which is a technique uses edge feature for face recognition, is a low computational cost and high accuracy technique in comparing with common methods for face recognition like Eigenface, LDA. Here, we proposed a modification of MHD method for face recognition. It is encouraging that the proposed method gives higher performance than MHD method in most of experiments.

In the following, a literature review of MHD method is given in section 2. Section 3 describes the modified of MHD method. In section 4, the performance of proposed method is examined for face recognition in various conditions of lighting, face expression and pose. Finally, the paper is concluded in section 5 .

\section{MHD METHOD FOR FACE RECOGNITION}

\subsection{Edge map of face image}

Edge, which is the reflecting of large intensity change that is caused by the geometrical feature of the object, is an important feature of object. Many edge extraction algorithms have been proposed and implemented. Heath et al. [9] compared the performance of different of edge detectors and had a conclusion that â€œno one 
single edge detector was best overall; for any given image it is difficult to predict which edge detector will be bestâ€国. In this paper, the edge detector based on the algorithm of Nevatia and Babu [10], followed with the thinning process is used for generating the one-pixel-width edge curve of a face image.

For reducing the number of points on the edge curves, Dynamic-two-strip (Dyn2S) is applied on edge curves for generating the dominant points of edge curves. In this paper, we will present a brief detail of Dyn2S algorithm. Futher information of Dyn2S algorithm could be found in [11]. In the Dyn2S algorithm, a strip is fitted to the left and right of each point on the curve, and the points inside each strip are approximated as a straight line. The orientation and width of the strip are adjusted automatically. The width of the strip could be adjusted from one tenth to equal the size of the image. The length of the strip is adjusted for covering as much points as possible. A measure of merit of each point could be calculated as $W=E^{\text {left }} . S . E^{\text {right }}$, where $S=\left|180^{\circ}-\theta\right|$ with $\theta$ is the angle between two strips, $E^{\text {left }}$ and $E^{\text {right }}$ are the ratio of the length and width of the left and right strip. The point with smaller merit in comparing with their neighbor is eliminated. The points that align approximately on a straight line are deleted except for the two endpoints on the curve. The remained points of edge curve are the dominant points. The result of applying Dyn2S algorithm on edge curves is edge map. An example of edge map of a face image is shown in Fig. 1.

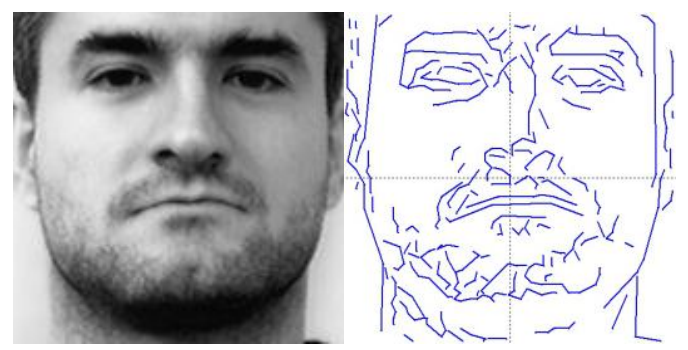

Fig. 1. A person face and its edge map.

\subsection{Modified Hausdorff distance (MHD)}

Hausdorff distance is a metric for measuring the distance between two point sets. Given two point sets $M=\left\{m_{1}, m_{2}, . ., m_{p}\right\}$, which represents the edge curve of a model in database, and
$T=\left\{t_{1}, t_{2}, \ldots, t_{q}\right\}$, which represents the edge curve of the test image. The original Hausdorff distance between two point sets is defined as:

$$
H(T, M)=\max (h(M, T), h(T, M))
$$

where

$$
h(M, T)=\max _{m_{i} \in M} \min _{t_{j} \in T}\left\|m_{i}-t_{j}\right\|
$$

and $\left\|m_{i}-t_{j}\right\|$ is the Euclidean distance of two points $m_{i}$ and $t_{j}$ as above definition, the Hausdorff distance $H(T, M)$ measures the degree of mismatch between the model and the test image. However, the Hausdorff distance as defined in Eq. (1) and (2) is sensitive to the outline points. If two face images have two edge curves with some outline points, or even one point, with the directed Hausdorff distance as Eq. (2), two edge curves might be quite similar.

Dubuisson and Jain [7] suggested a MHD for object matching, where the maximum distance of every points in the edge curve as Eq. (2) is replaced by the average distance. The MHD is defined as:

$$
h(M, T)=\frac{1}{P} \sum_{m_{i} \in M} \min _{t_{j} \in T}\left\|m_{i}-t_{j}\right\|
$$

where $P$ is the number of points in model edge curve.

Takács [3] used MHD to the edge curve of face for matching. However, the disadvantage of the MHD as TakÃ $i c s$ is the computational cost. If the model image has $P$ points and the test image has $Q$ points in the edge curve, the computational complexities of MHD will be $O(P Q)$. The number of points in each edge curve $P$ and $Q$ is very high.

Gao [8] proposed M2HD which overcome the disadvantage of MHD. Gao showed that it is not necessary to use all of points in the edge curve, some points with high curvatures on the edge curve could be a good presenting for edge curve. $\mathrm{He}$ called such points are the dominant points. So he applied M2HD algorithm for the edge map of face image. The M2HD is defined as: 


$$
H(T, M)=\max \left(h_{M^{2} H D}(M, T), h_{M^{2} H D}(T, M)\right)
$$

where

$$
h_{M^{2} H D}(M, T)=\frac{1}{\sum_{m_{i} \in M} W_{m_{i} t_{j}}} \sum_{m_{i} \in M} W_{m_{i} t_{j}} \cdot \min _{t_{j} \in T}\left\|m_{i}-t_{j}\right\|
$$

where $W_{m_{i} t_{j}}=1 / 2\left(W_{m_{i}}+W_{t_{j}}\right)$ is the average metric of two point $m_{i}$ and $t_{j}$, which are provided by Dyn2D algorithm.

The experiment results of M2HD by Gao [8] give the same performance as the MHD by TakÃ $\tilde{A}_{i c s}$ [3]. So the Hausdorff distance is calculated as Eq. (5) give the same result as calculated as Eq. (3). The contribution of Gao [8] is applying the Dyn2S for edge curve face, which called edge map, for reducing the number of point of edge curve. This makes lower storage and computational cost for system. Thus the MHD method for face recognition could be used as applying MHD method as Eq. (3) for the edge map of the face image.

\section{A MODIFICATION OF MHD METHOD FOR FACE RECOGNITION}

The MHD method as above still has the following weakness. Suppose $T$ is the set of dominant points in the edge map of the test image and $t_{j}$ is a dominant point in the edge map; $M_{c}$ is the corresponding identical model of $T$ in the database and $M_{n}$ is the nonidentical model of $T$ in the database. If the corresponding point of $t_{j}$ in the $M_{c}$ is missing because of the segmentation error or the noise, as the defining of MHD method, the point $t_{j}$ will take other nearest point $m_{c n} \in M_{c}$ as its corresponding point. The distance $d\left(t_{j}, m_{c n}\right)=\min _{m_{c} \in M_{c}} d\left(t_{j}, m_{c}\right)$ is used for the calculation in MHD method. Similarity, the nearest point $m_{n n} \in M_{n}$ of $t_{j}$ in $M_{n}$ is considered as the corresponding point and the distance $d\left(t_{j}, m_{n n}\right)$ is used for MHD calculation. It is possible that to have $d\left(t_{j}, m_{c n}\right) \gg d\left(t_{j}, m_{n n}\right)$ for matching the complicated and similar objecs as human face. This makes the mismatch for face identification.

The number of corresponding point between the input image and model image is another measure for similarity. The number of corresponding point between the input image and its corresponding model in the database should be larger than its non-corresponding model in the database. The problem as above mention could be alleviated by introducing the parameter of number corresponding point into the calculation of similarity.

Assume that $t_{j}$ is a point in the input edge map $T$ and $m_{i}$ is the corresponding point of $t_{j}$ in the model edge map $M . m_{i}$ should be the nearest point to $t_{j}$ in comparing with other points in $M$ and $m_{i}$ should be located near the point $t_{j}$ because the test image and the model have been aligned and scale normalized by preprocessing before matching. A position neighbor $N_{p}$ is introduced. If a point $m_{i}$ in the model edge map $M$ has the smallest distance the the point $t_{j}$ of the input edge map and $m_{i}$ locates within position neighbor of point $t_{j}$, such point $m_{i}$ names as high confident point. A high confident ratio of an image is defined as the ratio of the number of high confident point and total number point in the edge map.

$$
R=\frac{\text { numberofhighconfidentpoints }}{\text { numberoftotalpoints }}
$$

The disparity parameter between two images is defined as:

$$
D_{n}=1-\frac{R_{M}+R_{T}}{2}
$$

where $R_{M}$ and $R_{T}$ are the high confident ratio of the model edge map and input edge map.

The modified MHD (MMHD) is defined as:

$$
H_{M M H D}=\sqrt{H_{M H D}^{2}+\left(W_{n} * D_{n}\right)^{2}}
$$

where $H_{M H D}$ is the directed MHD as defined in section 2 at Eq. (3) and $W_{n}$ is the the weight of number disparity.

We use the face database [12] of Bern University for the training process for determining 
the parameters $\left(N_{p}, W_{n}\right)$. After training process, we get $N_{p}=0$ and $W_{n}=3$. Since this training process could be done offline, so it does not effect to the processing time of the MMHD method.

\section{EXPERIMENT RESULT}

In this section, the performance of the proposed method is investigated which all conditions of human face recognition. The proposed method is used for recognizing the human face under:

- Controlled condition - ideal condition.

- Varying lighting conditions.

- Varying head poses.

- Varying face expression.

In this paper, two databases were tested. The Bern University database [12], which is used for examining the system performance under ideal condition and head pose variation, contains images from 30 people which controlled lighting condition. Each person has 10 images: two frontal poses, two looking to the left, two looking to the right, two looking up and two looking down. The AR database [13] is used for examining the system performance under ideal condition, varying lighting conditions and varying face expressions. The AR database contains 2600 images of 100 people. Each person has 26 images which first 13 images with various lighting conditions, various face expressions and no restrictions on wear, hairstyle and make-up; last 13 images are the wo weeks later version of first 13 images. All images are normalized and cropped to size $160 \times 160$ pixels before matching process.

All of experiments were conducted on a PC station with $3.7 \mathrm{GHz} \mathrm{CPU}$ and $2 \mathrm{~GB}$ RAM. The results of MHD method are slightly different from the result of Gao in [8]. The reason is all of human face images in [8] are pre-processed better than the database used in this paper. In the paper of Gao, all of human face images are scaled and oriented for two eyes were aligned roughly at the same position with a distance of 80 pixels. In this paper, all of human images are scaled, without oriented, for the distance between two eyes were 80 pixels. The position of the eyes are not the same position. However, the pre-processing process has not strongly effect to the performance comparison between MHD and MMHD.

\subsection{Face recognition with ideal condition}

In this experiment, we use a pair of frontal face, which is the ideal conditions picture, of each person in the Bern database and AR database for face recognition. Examples of pair face is in Fig. 2 and Fig. 3. The recognition result is summarized in Table 1.

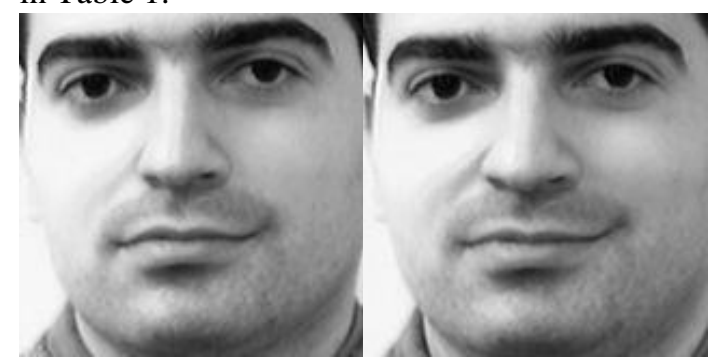

Fig. 2. A pair frontal face of Bern database.

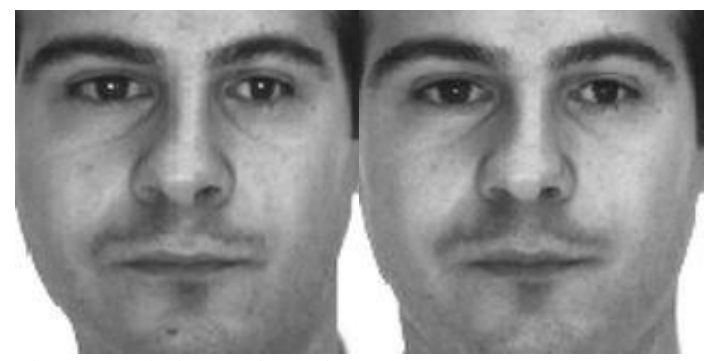

Fig. 3. A pair frontal face of AR database.

TABLE 1

RECOGNITION RATE FOR IDEAL CONDITION

\begin{tabular}{ccc}
\hline \hline Method & Bern & AR \\
\hline MHD & $100 \%$ & $69 \%$ \\
MMHD & $100 \%$ & $75 \%$ \\
\hline \hline
\end{tabular}

With Bern University database, all methods give correct recognition of $100 \%$ because there are minor differences between two frontal face pictures. However, two frontal face pictures of AR database is taken within 2 weeks, this makes large difference between two pictures. This makes the accuracy for AR database is lower than Bern database.

Here, the accuracy of the proposed method MMHD is $6 \%$ higher than MHD method for AR database. This is a reasonable improvement of the proposed method.

\subsection{Face recognition under varying lighting condition}

In this experiment, 100 frontal face pictures of AR database are used as the database. Each person has 2 pictures with the left-light, 2 pictures with the right-light and 2 pictures with the bothlight. So with each lighting condition, we have 200 pictures as the input. The face recognition under varying lighting condition result is showed 
in Table 2.

TABLE 2

RECOGNITION RATE UNDER VARYING LIGHTING CONDITIONS

\begin{tabular}{ccc}
\hline \hline Method & MHD & MMHD \\
\hline Left light on & $73 \%$ & $74 \%$ \\
Right light on & $66 \%$ & $68 \%$ \\
Both light on & $50 \%$ & $53 \%$ \\
\hline \hline
\end{tabular}

For all lighting conditions, the proposed method give higher accuracy, $1 \%$ to $3 \%$, than the MHD.

For real application, the lighting condition is not ideal. As the result of the proposed method as Table 2 and Table 1 for AR database, the accuracy is slightly stable with the lighting conditions. This makes MMHD is attractive for real applications.

\subsection{Face recognition with varying head poses}

In this experiment, frontal face images of 30 persons in Bern University database is used as the database. Each person has 2 pictures looking left, 2 pictures looking right, 2 pictures looking up and 2 pictures looking down. So we have 60 pictures for pose used as the input. The recognition result is shown in Table 3.

TABLE 3

RECOGNITION RESULT FOR VARYING HEAD POSES CONDITION

\begin{tabular}{ccc}
\hline \hline Method & MHD & MMHD \\
\hline Looking left & $55 \%$ & $57 \%$ \\
Looking right & $48 \%$ & $55 \%$ \\
Looking up & $60 \%$ & $47 \%$ \\
Looking down & $55 \%$ & $60.7 \%$ \\
Avarage & $54.5 \%$ & $54.8 \%$ \\
\hline \hline
\end{tabular}

The proposed method gives equal performance to MHD method in the average.

\subsection{Face recognition with varying face expressions}

In the experiment, we use 100 frontal face pictures of AR database for the database. Each person has 2 pictures of each face expression, so we have 200 pictures as input for each face expression experiment.

TABLE 4

RECOGNITION RESULT FOR VARYING FACE EXPRESSION

\begin{tabular}{ccc}
\hline \hline $\begin{array}{c}\text { Face } \\
\text { expression }\end{array}$ & MHD & MMHD \\
\hline Smiling & $70 \%$ & $72 \%$ \\
Angry & $85 \%$ & $85 \%$ \\
Sceaming & $20 \%$ & $25 \%$ \\
\hline \hline
\end{tabular}

For all human expressions, the proposed method give the same or higher accuracy, up to $5 \%$, than the MHD.

The screaming expression gives very low accuracy for face recognition. The reason is human face with screaming expression has too much different with human face in database, especial the mouth. However, the screaming expression is rarely appearance in real application. with the high accuracy for smiling and angry expression, the MMHD makes the application is friendly with user because of the free of face expressions.

\section{CONCLUSION}

MHD is an advantage method for face recognition in comparing with common method, Eigenface [8]. Especially, MHD is stable with the lighting condition, which is the most complicate problem for real application of face recognition, where the lighting condition is not ideal.

In this paper, a modification of MHD (MMHD) is proposed. By adding the high confident ratio in measuring the similarity between images, the proposed method gives higher performance than MHD method in all experiment.

The experiment results also shows that the proposed method attractive with real face recognition applications, where the lighting conditions or human face expression are not ideal.

\section{REFERENCES}

[1]L. Sirovich and M. Kirby, "Low-Dimensional Procedure for the Characterisation of Human Faces", J. Optical Soc. of Am., vol. 4, pp. 519-524, 1987.

[2]K. K. Sung and T. Poggio, "Learning Human Face Detection in Cluttered Scenes", Computer Analysis of Image and Patterns, pp. 432-439, 1995.

[3]B. Takács, "Comparing Face Images Using the Modified Hausdorff Distance", Pattern Recognition, vol. 31, 1998, pp. 1873-1881.

[4]M. Lades et al., "Distortion Invariant Object Recognition in the Dynamic Link Architecture", IEEE Trans. Computers, vol. 42, pp. 300-311, 1993.

[5]F. Samaria and F. Fallside, "Face Identification and Feature Extraction Using Hidden Markov Models", Image Processing: Theory and Application, G. Vernazza, ed., Elsevier, 1993.

[6]B. S. Manjunath, R. Chellappa, and C. von der Malsburg, "A feature based approach to face recognition", in Proceedings 1992 IEEE Computer Society Conference on Computer Vision and Pattern Recognition, pp. 373-378, Jun 1992.

[7]M. P. Dubuisson and A. K. Jain, "A modified Hausdorff distance for object matching", Proc. 12th Int. Conf. on Pattern recognition, pp. 566-568, Jerusalem, Israel, 1994.

[8]Y. Gao, "Efficiently comparing face images using a modified Hausdorff distance", IEEE Proc. Vision, Image 
and Signal Processing, vol. 150, no. 6, Dec. 2003, pp. 346-350.

[9]M. D. Heath, S. Sarkar, T. Sanocki, and K. W. Bowyer, "Comparison of edge detectors: a methodology and initial study”, Comput. Vis. Image Underst., vol. 69, 1998, pp. $38-54$.

[10]R. Nevatia, and K. R. Babu, "Linear feature extraction and description”, Comput. Graph. Image Process., vol. 13, pp. 257-269, 1980.

[11]M. K. H. Leung and Y. H. Yang, "Dynamic two-strip algorithm in curve fitting", Pattern Recognition, vol. 23, 1990, pp. 69-79.

[12]Bern University Face Database, http://www.fki.inf.unibe.ch/databases/iam-faces-database, 2002.

[13]A. M. Martinez and R. Benavente, "The AR Face Database”, CVC Technical Report \#24, June 1998.

Dang Nguyen Chau received the B.S. degree in electrical engineering from the Ho Chi Minh City University of Technology, VNU-HCM, Vietnam, in 2010 and the M. Eng. degree in electrical, electronic and information engineering from the Toyohashi University of Technology, Japan in 2013. Since 2011 he has been with the Faculty of
Electrical and Electronics Engineering, Ho Chi Minh City University of Technology, VNU-HCM, Vietnam. His research interests include iamge processing, computer vision and machine learning.

Do Hong Tuan received the B.S. and M. Eng. degrees in electrical engineering from the Ho Chi Minh City University of Technology, VNU-HCM, Vietnam, in 1994 and 1997, respectively, the M.Sc. and $\mathrm{Ph}$. D. degrees in communication engineering from the Munich University of Technology, Germany, in 2000 and 2004, respectively. Since 1994 he has been with the Faculty of Electrical and Electronics Engineering, Ho Chi Minh City University of Technology, VNU-HCM, Vietnam. His research interests include stochastic signal processing and applications for communications, image and video.

\title{
Cải tiến tốc độ tính toán cho phương pháp khoảng cách Hausdorff đã hiệu chỉnh dùng cho nhận dạng khuôn mặt
}

\author{
Đặng Nguyên Châu và Đỗ Hồng Tuấn
}

\begin{abstract}
Tóm tắt- Nhận dạng khuôn mặt có rất nhiều ứng dụng trong cuộc sống hiện nay nên nó vẫn là một hướng nghiên cứu thu hút của cộng động nghiên cứu về nhận dạng. Vì sự giống nhau của khuôn mặt nên vấn đề nhận dạng khuôn mặt với độ chính xác cao vẫn còn là một thách thức với các nhà nghiên cứu. Phương pháp khoảng cách Hausdorff đã hiệu chỉnh (MHD) dựa trên khoảng cách Hausdorff là một phương pháp đơn giản nhưng có độ chính xác rất cao trong nhận dạng khuôn mặt. Trong bài báo này, một cải tiến của phương pháp MHD được đề xuất. Bằng cách thêm tỷ số độ tin cậy vào việc tính khoảng cách giữa các bức ảnh, phương pháp đề xuất đã cho kết quả nhận dạng cao hơn so với phương pháp cũ MHD. Phương pháp đề xuất cũng cho kết quả cao hơn phương pháp MHD trong việc nhận dạng khuôn mặt với các điều kiện không lý tưởng về ánh sáng, góc chụp khuôn mặt và cảm xúc khuôn mặt trên bức ảnh cần nhận dạng.
\end{abstract}

Tù khóa — Nhận dạng khuôn mặt; bản đồ cạnh; khoảng cách Hausdorff; MHD. 